\title{
Traduire
}

Une autre perspective sur r tr traduction

Revue française de la traduction

245 | 2021

Regards sur l'interprétation

\section{Portrait-robot de l'interprète. Quelques réflexions personnelles}

\section{Christine Cross}

\section{Q OpenEdition \\ 1 Journals}

\section{Electronic version}

URL: https://journals.openedition.org/traduire/2407

DOI: 10.4000/traduire.2407

ISSN: 2272-9992

\section{Publisher}

Société française des traducteurs

\section{Printed version}

Date of publication: 5 December 2021

Number of pages: 5-10

ISSN: 0395-773X

\section{Electronic reference}

Christine Cross, "Portrait-robot de l'interprète. Quelques réflexions personnelles", Traduire [Online], 245 2021, Online since 05 December 2021, connection on 23 December 2021. URL: http:// journals.openedition.org/traduire/2407 


\title{
Portrait-robot de l'interprète
}

\author{
Quelques réflexions personnelles
}

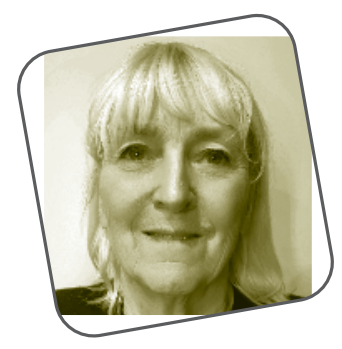

\section{Christine Cross}

L'interprétation en tant que telle existe de manière ad hoc depuis des lustres, mais ce n'est qu'au cours du siècle dernier que la profession a commencé à sortir de sa chrysalide. D'abord exercée par des personnes bilingues ou polyglottes naturellement douées pour la transposition d'une langue à l'autre, elle a ensuite été projetée sur le devant de la scène par les grands événements politiques $d u x x^{e}$ siècle (guerres, déplacements de personnes...).

Ces phénomènes, associés à ceux de l'évolution technologique, ont poussé certains grands interprètes de l'époque à créer des écoles d'interprétation, la toute première étant celle de Genève (Suisse) fondée en 1941. L'Allemagne lui a emboité le pas en 1946, suivie de la France en 1948 et de I'Autriche en 1952'. Les bases étaient jetées: la formation structurée des interprètes allait enfin devenir une réalité.

À l'heure actuelle, l'interprétation vit une mini-révolution, boostée par la pandémie de Covid-19. Pourtant, même si 
la technologie d'aujourd'hui permet de pratiquer le métier à distance, à partir d'une cabine équipée ou du domicile de l'exécutant, les qualités essentielles du bon interprète demeurent inchangées...

Quelles sont donc ces aptitudes, innées ou acquises, qui font d'un linguiste compétent un bon interprète?

Beaucoup d'entre-elles vont sans dire: une très bonne connaissance de sa langue maternelle, une grande maîtrise d'une ou de plusieurs langues étrangères, une très bonne mémoire et une vivacité d'esprit sans faille. D'autres sont peut-être moins évidentes (passion de la communication, diction impeccable, maîtrise du débit, capacité d'analyse...).

De prime abord, il peut paraitre inutile d'insister sur la connaissance de sa langue maternelle mais si l'interprète ne sait pas bien formuler des phrases dans cette langue et commet des fautes de grammaire ou d'accord, ses interlocuteurs vont vite se poser des questions sur son professionnalisme. Et puis, bien manier sa langue permet d'en jover comme d'un instrument pour mieux communiquer l'essence du message à traduire. Ce n'est pas par hasard que le terme «interprète» s'applique aussi bien à celui ou celle qui joue d'un instrument qu'à celui ou celle qui s'exprime à l'aide de ses cordes vocales. En outre et de toute évidence, une voix bien modulée sera beaucoup plus agréable à écouter qu'une voix plate, stridente ou tellement douce qu'on a du mal à l'entendre, tout comme un instrument de musique bien accordé produira toujours une sonorité supérieure.

Inutile également, me direz-vous, de parler de la maîtrise des langues étrangères. Comme dans le cas de sa propre langue, il n'est pas simplement question de posséder un vocabulaire riche et une bonne compréhension. II convient aussi de s'immerger dans la culture du ou des pays de ses autres langues de travail. Lorsqu'ils interviennent dans le cadre de grandes manifestations internationales, les orateurs ont tendance à puiser leurs exemples dans la culture de leur pays d'origine, à citer des références typiques de ce pays, à appuyer leur discours sur une expérience profondément teintée de leur propre vécu.

Ce phénomène peut poser problème pour l'interprète. Je me rappelle avoir participé à un congrès mondial se déroulant au moment où la série télévisée britannique Docteur 
Who ${ }^{2}$ fêtait son $40^{\circ}$ anniversaire. Cette émission, culte en Grande-Bretagne, n'a pas soulevé les mêmes passions de l'autre côté de la Manche où elle est bien moins connue. Heureusement pour moi, je venais de lire un article dans la presse britannique qui parlait de cette série de sciencefiction dont le héros éponyme voyage à travers le temps dans un vaisseau ressemblant à une cabine téléphonique (appelée TARDIS), car j'étais alors tenue d'interpréter le discours d'un intervenant anglophone ayant choisi de comparer sa profession à ce vaisseau dont la caractéristique première est d'être infiniment plus grand à l'intérieur qu'à l'extérieur. Sans cette lecture fortuite, je ne sais pas trop comment j'aurais pu trouver un sens à cette référence! D'où la nécessité d'une très grande curiosité et d'une capacité à absorber même les faits les plus anodins ou les plus saugrenus!

L'interprète doit être curieux de tout: ce n'est pas parce que vous allez interpréter lors d'un congrès de médecins qu'on ne parlera pas du Tour de France ou des Jeux olympiques. Des connaissances en histoire, en géographie, en science, en technologie... vous seront toujours utiles. II convient de maintenir à jour ses bagages culturels. Une évidence? Logiquement, oui, mais nombreuses ont été les fois où j'ai constaté que l'interprète avait raté son rendez-vous avec l'actualité. Je n'ai qu'à penser aux interprétations en direct à la télévision, française en l'occurrence, pour me rappeler certains moments où l'interprète a probablement connu un grand moment de solitude!

Quid maintenant de la passion de la communication? Par définition, interpréter c'est permettre aux uns de comprendre les autres, établir une relation entre eux, faciliter des négociations commerciales voire politiques, participer à des échanges de savoirs ou de connaissances. Tandis qu'en mode simultané, l'interprétation se pratique à distance, autrefois à partir d'une cabine et désormais de plus en plus souvent depuis chez soi, en mode consécutif, elle met l'interprète en prise directe avec ses clients. Il faut alors savoir gérer le relationnel, créer un climat de confiance avec ses différents interlocuteurs, les mettre à l'aise.

2. https://fr.wikipedia.org/wiki/Doctor_Who 
J'ai également fait mention de la diction, de la bonne prononciation, de la nécessité de bien énoncer ses propos. À une époque où on a tendance à mettre en avant les accents régionaux, il est à contre-courant, mais nécessaire, d'insister sur les avantages d'un accent neutre, d'une prononciation claire et d'un discours dépourvu d'expressions régionales ou élitistes que pourraient ne pas comprendre les personnes non natives. Quand on intervient sur la scène internationale, on doit tenir compte des capacités de son public cible, bien choisir son vocabulaire, bien maîtriser son débit et bien moduler sa voix.

Une bonne mémoire revêt également toute son importance. Ne jamais perdre le fil de l'échange ou la suite de vos idées, bien retenir les noms et les chiffres, savoir prendre des notes et restituer les propos de l'orateur. Conjuguée à une bonne capacité d'analyse, la mémoire est un outil sans prix. Et cela s'entraîne. N'oublions pas la nécessité d'une bonne présence d'esprit, de la capacité à se contenter d'une solution qui «passe» (un autre terme ou une périphrase juste) lorsque, dans le feu de l'action, le mot parfait vous échappe. Un traducteur pourra toujours remettre son travail sur le métier tant qu'il n'en sera pas satisfait. L'interprète n'a que quelques secondes pour trouver une pirovette et se tirer d'affaire s'il ne tombe pas tout de suite sur l'expression idéale. Il doit également toujours garder son calme.

Quand je passe en revue le profil des interprètes que j'ai pu côtoyer professionnellement au cours de ma (longue) carrière, je me dois de constater que les meilleurs avaient grosso modo le même profil. Hormis leurs connaissances linguistiques, ils étaient tous des amateurs passionnés de la langue. Certains étaient très doués pour faire des jeux de mots, des vanneurs de haut vol, ils faisaient rapidement le lien entre les propos prononcés et le sens profond qui les sous-tendait. En cabine, il leur arrivait parfois de finir leur phrase avant l'intervenant lui-même, tellement ils s'étaient immergés dans le discours.

Quant aux jeunes fraîchement sortis de l'école supérieure avec leur diplôme de master d'interprétation en poche qu'il nous arrivait d'embaucher pour venir renforcer notre équipe (à l'époque où j'étais salariée au sein d'une organisation internationale), on sentait immédiatement lesquels ne 
dépasseraient jamais le stade de la simple compétence et lesquels dégageaient déjà un parfum de star.

En conclusion, si vous avez envie de vous lancer dans le monde de l'interprétation au $x \times I^{e}$ siècle, sachez que vous aurez tout intérêt à vous familiariser avec les nouvelles technologies qui vont de plus en plus influer sur la façon d'exercer le métier d'interprète. Vous ne devrez jamais vous dire que vous connaissez vos différentes langues à la perfection. Les langues évoluent sans arrêt, les mots qui prévalent aujourd'hui pourront céder leur place à d'autres dans les mois et les années à venir. Nos récentes expériences avec la pandémie de Covid-19 nous ont bien rappelé que la capacité à créer de nouveaux mots ne manque pas (pass sanitaire, distanciation sociale, cas contact, événements en présentiel ou en distanciel...).

Inscrivez-vous dans une des écoles proposant un master d'interprétation. Là, vous pourrez parfaire vos connaissances linguistiques, apprendre les astuces pour resserrer votre discours, vous frotter à des professionnels qui vous encadreront et vous aideront à trouver vos marques, réaliser des stages en immersion dans la mesure où vous obtiendrez l'une des rares places disponibles, attribuées en fonction d'un tri très strict.

Vous y apprendrez également comment aiguiser vos techniques d'interprétation, la prise de notes par exemple. Grâce à la pratique, vous affinerez votre capacité d'analyse. En même temps, vous serez en train de tisser les premiers liens de votre futur réseau.

L'assiduité est aussi un élément clé: lisez tous les documents de travail que les organisateurs d'événements pourront vous transmettre. II ne faut pas lésiner sur les préparatifs, sur les recherches. Aujourd'hui, avec internet, les apprentis interprètes disposent de moyens d'investigation dont n'ont pu bénéficier leurs aînés. Préparez-vous bien et ne prenez jamais rien pour acquis.

Si je devais brosser le portrait du ou de la candidat(e) idéal(e) à une carrière d'interprète, je décrirais quelqu'un de confiant mais pas trop, pas au point de ne jamais douter de lui ou d'elle-même. Un bon interprète est comme un comédien: il va toujours ressentir un petit trac avant son entrée 
en scène. D'ailleurs, interpréter c'est également, en quelque sorte, camper des personnages différents!

Ensuite, la personne aurait un bon relationnel, saurait comment s'imposer au milieu des bruits parasites sans crier trop fort, et aurait une tonalité de voix rassurante de nature à inspirer la confiance parmi ses auditeurs.

En revanche, si vous êtes timide, si vous avez horreur de l'approximatif, si vous risquez de perdre pied en cas de raté, je vous conseillerais de trouver votre créneau dans un domaine mieux adapté à votre personnalité.

Le cerveau est un muscle et, quel que soit votre âge, vous devez le faire travailler. Si vous vous lancez dans le monde de l'interprétation, rappelez-vous que vous aurez besoin de ce muscle tout au long de votre carrière professionnelle. Prenez donc bien soin de lui: il vous le revaudra bien sans aucun doute.

\section{cross.christine@gmail.com}

Christine CROSS est une traductrice-interprète anglophone désormais en fin de carrière. Elle a fait partie de la Commission "Traduire» pendant une dizaine d'années et a enseigné différentes disciplines à I'ISIT (traduction vers l'anglais, prise de parole professionnelle, tutorats...). Elle a réalisé l'essentiel de sa carrière dans le domaine du transport (salariée dans une organisation internationale) et, plus tard, dans celui de l'archivage (en freelance). Elle a également travaillé dans la communication multilingue auprès d'une grande entreprise multinationale (rédaction de publications institutionnelles, de lettres d'information, de communiqués de presse, etc. en six langues, à l'aide de ses correspondants dans les différents pays). 